\title{
C3 glomerulopathy-associated CFHR1 mutation alters FHR oligomerization and complement regulation
}

\author{
Agustín Tortajada,1,2 Hugo Yébenes, ${ }^{1}$ Cynthia Abarrategui-Garrido,,2,3 Jaouad Anter,,1,2 \\ Jesús M. García-Fernández,1,2 Rubén Martínez-Barricarte,1,2 María Alba-Domínguez,2,3 \\ Talat H. Malik, ${ }^{4}$ Rafael Bedoya, ${ }^{5}$ Rocío Cabrera Pérez, ${ }^{5}$ Margarita López Trascasa, ${ }^{2,6}$ \\ Matthew C. Pickering, ${ }^{4}$ Claire L. Harris, ${ }^{7}$ Pilar Sánchez-Corral,2,3 \\ Oscar Llorca, ${ }^{1}$ and Santiago Rodríguez de Córdoba ${ }^{1,2}$
}

${ }^{1}$ Centro de Investigaciones Biológicas, Consejo Superior de Investigaciones Científicas, Madrid, Spain.

${ }^{2}$ Centro de Investigación Biomédica en Enfermedades Raras, Madrid, Spain. ${ }^{3}$ Unidad de Investigación, Hospital Universitario de La Paz/IdiPAZ, Madrid, Spain. ${ }^{4}$ Centre for Complement and Inflammation Research, Imperial College, London, United Kingdom. ${ }^{5}$ Servicios de Nefrología Pediátrica y Anatomía Patológica, Hospital Virgen de Rocío, Sevilla, Spain. ${ }^{6}$ Unidad de Inmunología, Hospital Universitario de La Paz/IdiPAZ, Madrid, Spain.

${ }^{7}$ Institute of Infection and Immunity, School of Medicine, Cardiff University, Cardiff, United Kingdom.

\begin{abstract}
C3 glomerulopathies (C3G) are a group of severe renal diseases with distinct patterns of glomerular inflammation and $\mathrm{C} 3$ deposition caused by complement dysregulation. Here we report the identification of a familial C3G-associated genomic mutation in the gene complement factor $\mathrm{H}$-related 1 (CFHR1), which encodes FHR1. The mutation resulted in the duplication of the $\mathrm{N}$-terminal short consensus repeats (SCRs) that are conserved in FHR2 and FHR5. We determined that native FHR1, FHR2, and FHR5 circulate in plasma as homo- and hetero-oligomeric complexes, the formation of which is likely mediated by the conserved $\mathrm{N}$-terminal domain. In mutant FHR1, duplication of the N-terminal domain resulted in the formation of unusually large multimeric FHR complexes that exhibited increased avidity for the FHR1 ligands C3b, iC3b, and C3dg and enhanced competition with complement factor $\mathrm{H}(\mathrm{FH})$ in surface plasmon resonance (SPR) studies and hemolytic assays. These data revealed that FHR1, FHR2, and FHR5 organize a combinatorial repertoire of oligomeric complexes and demonstrated that changes in FHR oligomerization influence the regulation of complement activation. In summary, our identification and characterization of a unique CFHR1 mutation provides insights into the biology of the FHRs and contributes to our understanding of the pathogenic mechanisms underlying C3G.
\end{abstract}

\section{Introduction}

The family of factor H-related proteins (FHRs) includes 5 plasma proteins, FHR1-FHR5, with concentrations ranging 5-50 $\mu \mathrm{g} / \mathrm{ml}$, that are structurally and functionally related to factor $\mathrm{H}(\mathrm{FH})$, the main regulator of the alternative pathway of complement activation (1). The genes encoding these FHRs, CFHR1-CFHR5, are located downstream and are closely linked to the $\mathrm{FH}$ gene $(\mathrm{CFH})$, from which they likely originated by tandem duplication events (ref. 2 and Supplemental Figure 1; supplemental material available online with this article; doi:10.1172/JCI68280DS1). Although it is generally accepted that the FHRs influence complement activation and regulation via their interaction with $\mathrm{C} 3 \mathrm{~b}, \mathrm{iC} 3 \mathrm{~b}$, and $\mathrm{C} 3 \mathrm{dg}$ fragments and carbohydrates on surfaces, how this occurs remains controversial.

All FHRs retain the C-terminal region of FH (Supplemental Figure 1), with different degrees of sequence conservation, which explains their ability to interact with C3b, iC3b, C3dg, and carbohydrates $(1,3,4)$ and justifies the concept that FHRs compete with $\mathrm{FH}$ for binding to specific surfaces, enhancing alternative pathway (AP) activation. Weak cofactor and/or decay-accelerating activities have also been reported for some of the FHRs (3-7), suggestive of intrinsic complement regulatory activity.

Authorship note: Agustín Tortajada, Hugo Yébenes, and Cynthia Abarrategui-Garrido contributed equally to this work.

Conflict of interest: The authors have declared that no conflict of interest exists.

Citation for this article: J Clin Invest. 2013;123(6):2434-2446. doi:10.1172/JCI68280.
The CFH-CFHR1-CFHR5 gene region shows significant genetic variability that has been repeatedly associated with pathology (reviewed in ref. 8). In addition to conventional polymorphisms due to sequence variations, the presence within this region of large genomic duplications (ranging $1.2-38 \mathrm{~kb}$ in size) makes the region highly prone to genomic rearrangements through gene conversion and nonhomologous recombination events (2). Many of these CFH-CFHR1-CFHR5 gene rearrangements have been associated with a number of chronic and infectious diseases. Interestingly, deletion of the CFHR1 and CFHR3 genes $\left(\Delta_{\text {CFHR3-CFHR1 }}\right)$, a common polymorphism in humans, is associated with lower risk for agerelated macular degeneration (AMD) (9) and IgA nephropathy (10), whereas generation of mutant FHRs by gene fusion or internal duplication events has been associated with increased risk for kidney diseases, like atypical hemolytic uremic syndrome (aHUS) and C3 glomerulopathy (C3G) (11-13).

$\mathrm{C} 3 \mathrm{G}$ is a heterogeneous group of kidney pathologies associated with complement dysregulation and classified histologically by C3 predominant glomerulonephritis. In C3G of the dense deposit disease type (DDD), the dense material appears within the glomerular basement membrane (GBM), whereas in C3 glomerulonephritis (C3-GN), it is predominantly mesangial and/or subendothelial in location (14). Phenotypes that are intermediate between DDD and C3-GN also occur.

We report here the identification of a mutant FHR1 protein associated with $\mathrm{C} 3 \mathrm{G}$ that highly resembled the mutant FHR5 
GN29

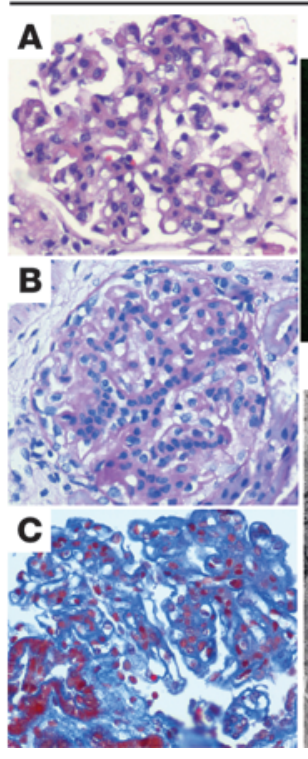

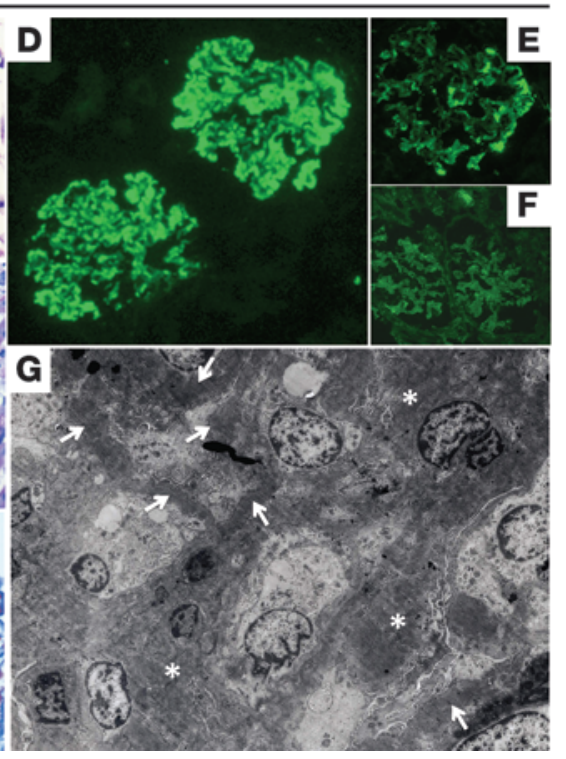
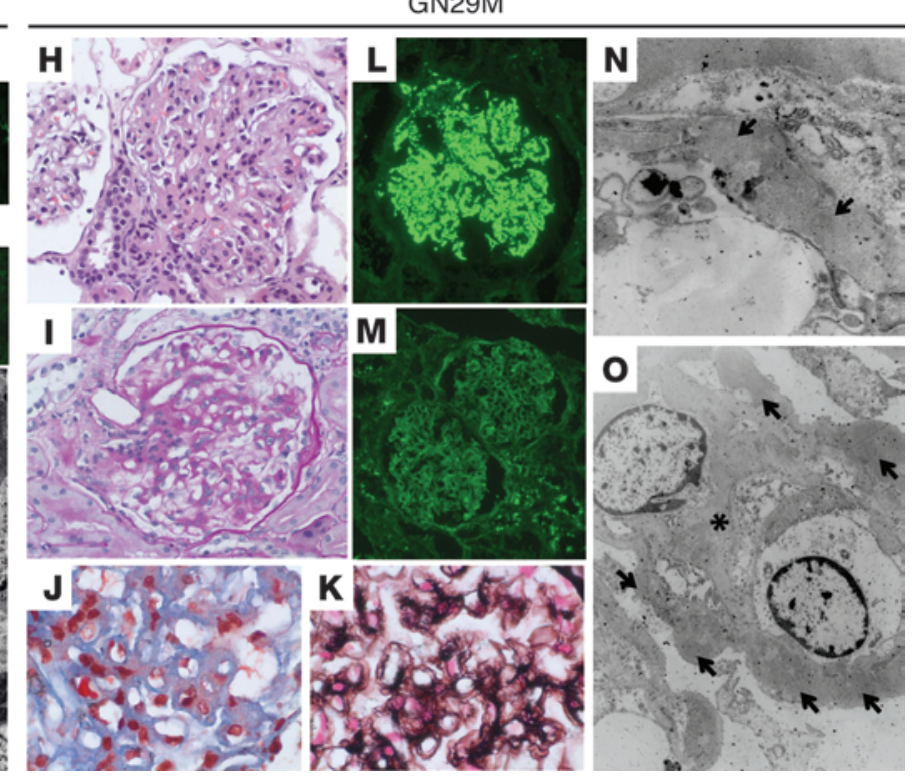

Figure 1

Histology, immunofluorescence, and EM findings. Kidney biopsies from probate GN29 (A-G) and his mother, GN29M (H-O), showed remarkably similar light, immunofluorescence, and ultrastructural findings. The characteristic histological lesion consisted of mesangial hypercellularity with thickened, eosinophil-rich segments of GBM (A and $\mathbf{H})$. The affected glomerular segments were PAS positive and reacted to trichrome and Jones methenamine-silver stain (B, C, and $\mathbf{I}-\mathbf{K})$. The main immunofluorescence findings were prominent and diffuse $\mathbf{C} 3$ deposits, which were granular in some glomerular areas ( $\mathbf{D}$ and $\mathbf{L})$. IgG was absent from these deposits (F and $\mathbf{M})$, although local deposits of IgM were observed in $\mathbf{G N 2 9}(\mathbf{E})$. Both biopsies showed similar ultrastructural alterations (G, N, and $\mathbf{O}$ ) consisting of the presence of ribbon-like, osmiophilic deposits in the GBM (arrows); these electron-dense deposits were also evident in the mesangial matrix (asterisks). Original magnification, $\times 400$ (A-C, H, and I); $\times 200$ (D-F); $\times 1,600$ (G); $\times 600(\mathbf{J}$ and $\mathbf{K}) ; \times 100(\mathbf{L}$ and $\mathbf{M}) ; \times 2,950(\mathbf{N}) ; \times 8,900(\mathbf{O})$.

protein, with an internal duplication of short consensus repeat 1 (SCR1) and SCR2 that has been associated with familial C3-GN among individuals with Cypriot ancestry (15). It also resembled the hybrid FHR2::FHR5 protein reported by Chen et al. in a DDD pedigree, in which SCR1 and SCR2 of FHR2 are fused to the fulllength FHR5 protein (11). A striking common characteristic of these 3 mutant FHRs associated with C3G is the duplication of the $2 \mathrm{~N}$-terminal SCRs that are virtually identical in the FHR1, FHR2, and FHR5 proteins (Supplemental Figure 1). The inheritance pattern of these mutations as a dominant trait underlies the functional relevance of the $\mathrm{N}$-terminal region of these FHRs and suggests a gain-of-function outcome.

Based on these data, we hypothesized that characterization of the structural organization of the human proteins FHR1, FHR2, and FHR5 in plasma would be critical for understanding the functional consequences of the mutant FHR1 protein. Our data demonstrated that FHR1, FHR2, and FHR5 circulated in plasma as homo- and hetero-oligomeric complexes and suggested that the $\mathrm{N}$-terminal SCR1 and SCR2 are involved in the oligomerization. The structural organization of FHR1, FHR2, and FHR5 in plasma helps to understand how these proteins influence complement activation and regulation. Crucially, multimerization as a consequence of duplication of the oligomerization domains in the mutant FHR1 protein increased binding to $\mathrm{C} 3 \mathrm{~b}$, iC $3 \mathrm{~b}$, and $\mathrm{C} 3 \mathrm{dg}$ and resulted in enhanced competition with FH. Taken together, these structural and functional data explain how mutant FHR1 causes complement dysregulation and reveal a novel pathogenic mechanism of C3G.

\section{Results}

A familial case of $C 3 G$ associated with an internal duplication of CFHR1. We present the case of a 12-year-old boy (GN29), his mother (GN29M), and his maternal grandfather (GN29GF); see Methods for detailed clinical presentation and development of disease. Biopsies in all showed C3G (Figure 1). Ultrastructural changes were heterogeneous: in GN29, deposits were present within the GBM and mesangium; in GN29M, accumulation of electron-dense material was found in subendothelial and subepithelial GBM locations (Figure 1).

We were able to collect samples from the 2 affected members alive in the pedigree, GN29 and GN29M, and from 2 healthy relatives, GN29B and GN29U (Figure 2A). Complement analysis in the proband and his mother illustrated persistent decreased levels of C3 compared with their healthy relatives (Figure 2B), which suggests significant activation of complement. Notably, C3 levels in these patients, although decreased, were significantly higher than those usually found in DDD patients. Assays to identify C3Nef or anti-FH autoantibodies were negative. Levels of membrane cofactor protein (MCP; also known as CD46) on the surface of peripheral blood lymphocytes were normal in both patients. Levels of factor I (FI), factor B (FB), and FH were also normal.

Routine Western blot analysis to characterize anomalies in the FHRs, performed as previously described (16), identified an anomalous FHR band of approximately $70 \mathrm{kDa}$ in the proband and his mother that was absent in their healthy relatives (Figure 2C). Proteomic analysis of material from this band revealed that it exclusively contained sequences corresponding to FHR1 (Supplemental Figure 2). Multiplex ligation-dependent probe 


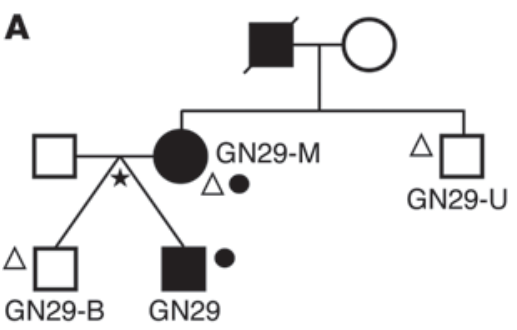

- Mutant FHR1

$\triangle \Delta_{\text {CFHR3-CFHR1 }}$

$\star$ IVF, nonidentical twins
B

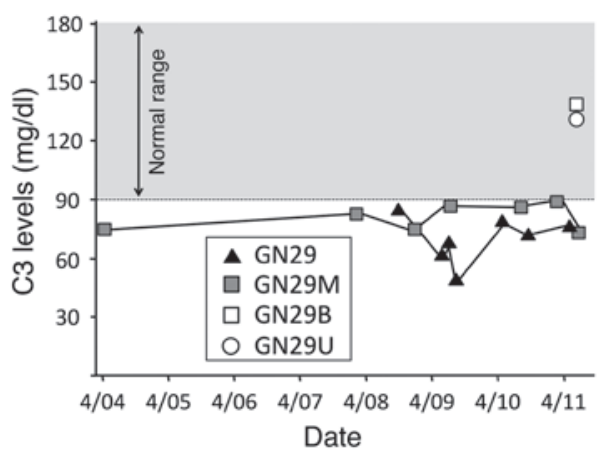

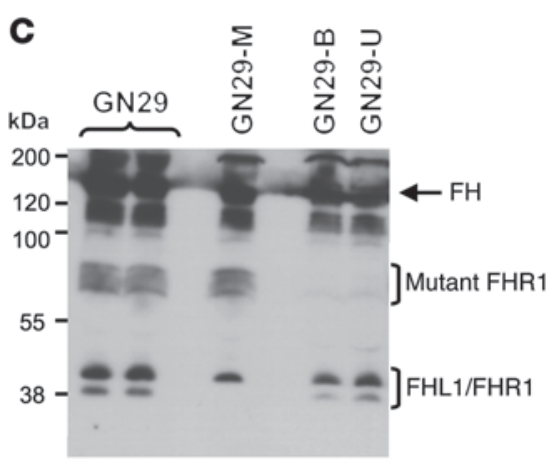

D

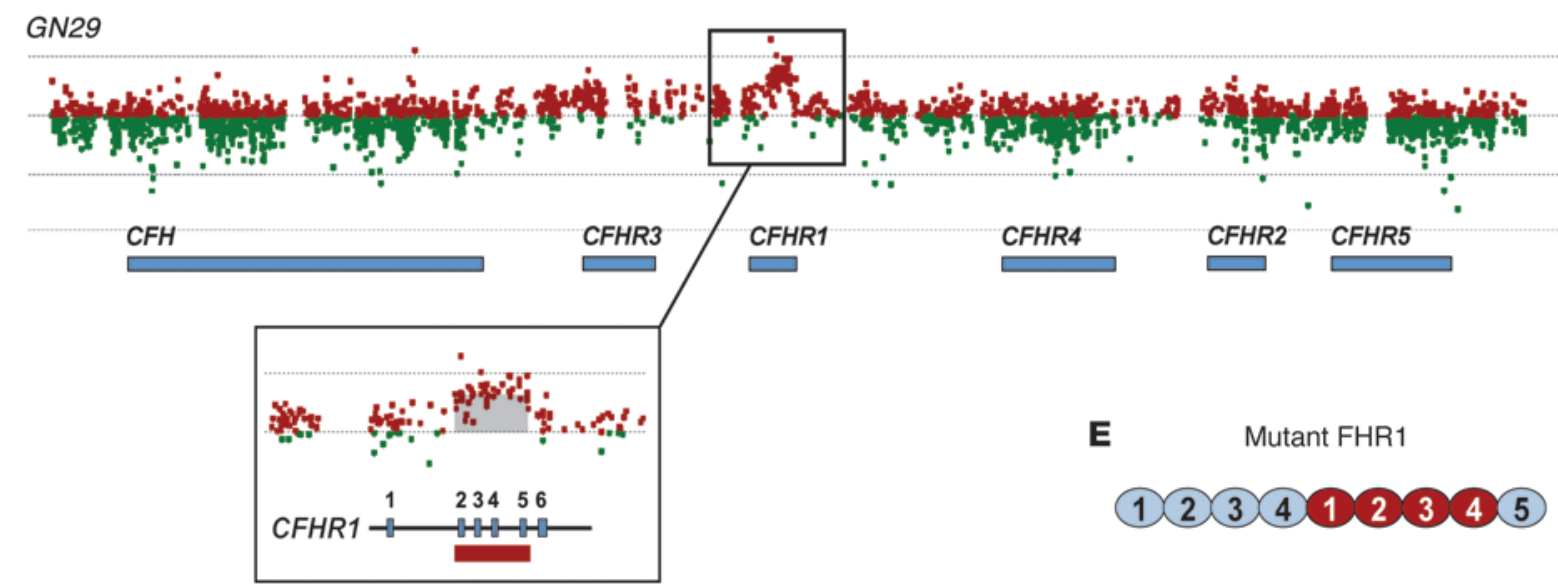

Figure 2

Internal duplication of CFHR1 is associated with C3G in pedigree GN29. (A) The GN29 pedigree. Affected individuals are indicated with solid symbols; carriers of the mutant FHR1 and of $\triangle_{\text {CFHR3-CFHR1 }}$ are indicated with solid circles and triangles, respectively. (B) Levels of C3 in affected and healthy individuals in the GN29 pedigree. (C) Western blot of whole human plasma identifying FH, FHL1, and FHR1 with an in-house rabbit polyclonal anti-FH. An anomalous band of approximately $70 \mathrm{kDa}$ was identified in the proband and his mother. Proteomic analysis of material from this band demonstrated that this band was the product of a mutant CFHR1 gene (Supplemental Figure 2). (D) High-resolution CGH array of the CFH-CFHR1 locus, illustrating an internal duplication of CFHR1 in the genomic DNA from GN29, encompassing exons 2-5. (E) Putative structure of a 9-SCR protein encoded by the mutant CFHR1. Red denotes duplicated SCRs.

amplification (MLPA) analysis identified 2 rearrangements in the CFHR1-CFHR5 region segregating in this pedigree, the polymorphic deletion of the CFHR3 and CFHR1 genes $\left(\Delta_{\text {CFHR3-CFHR1 }}\right)$ and a partial duplication of the CFHR1 gene, which was only carried by the affected individuals (Supplemental Figure 3A) and likely explains the anomalous FHR1 band found by Western blot in these individuals. To further characterize this CFHR1 genomic rearrangement, genomic DNA from the proband was analyzed in a custom-made high-density comparative genomic hybridization (CGH) $15 \mathrm{k}$ array (see Methods), which was designed to identify copy number variations within the regulators of complement activation (RCA) gene cluster. These analyses revealed an internal duplication of exons 2-5 in CFHR1 that predicted a mutant FHR1 protein (Figure 2D), consistent with the anomalous FHR1 band detected in the proband and his affected mother. Long PCR amplification analyses using primers to exons 5 and 2 of CFHR 1 resulted in the generation of DNA fragments that confirmed the fusion of intron 5 to intron 1 (Supplemental Figure 3B). As a whole, these data demonstrate that in pedigree GN29, the disease segregates with an anomalous mutant FHR1 protein resulting from an internal duplication of the CFHR1 gene. This protein had 9 SCRs, with the first 4 duplicated in tandem (Figure 2E). Thus, the mutant FHR1 protein was expressed at concentrations similar to those of the native FHR1 protein and could be easily detected in the plasma of the mutation carriers.

The mutant FHR1 protein resembles the mutant FHR5 originally described by Gale et al. in Cypriots (14), and the FHR2::FHR5 hybrid protein described by Chen et al. (11), in the sense that each of these 3 mutant proteins is characterized by the duplication of SCR1 and SCR2. In all cases, these aberrant proteins were associated with C3G, either DDD or C3-GN. Interestingly, the disease showed full penetrance in the carriers of these 3 mutations. It also segregated as a dominant trait, which suggests that, in contrast to the protective effect of the $\Delta_{\text {CFHR3-CFHR1 }}$ allele, duplication of the N-terminal region of these FHRs results in a gain of function.

Native FHR1 interacts with FHR2 and FHR5, but not with FH, FHR3, or $F H R 4 A / B$. To understand the functionality of the $\mathrm{N}$-terminal SCR1 and SCR2 domains of FHR1 and to determine the consequences of the duplication of this region in the FHR1 protein, we purified native FHR1 from FH-depleted human EDTA plasma using, as a first step, heparin chromatography with a 50- to 350- 

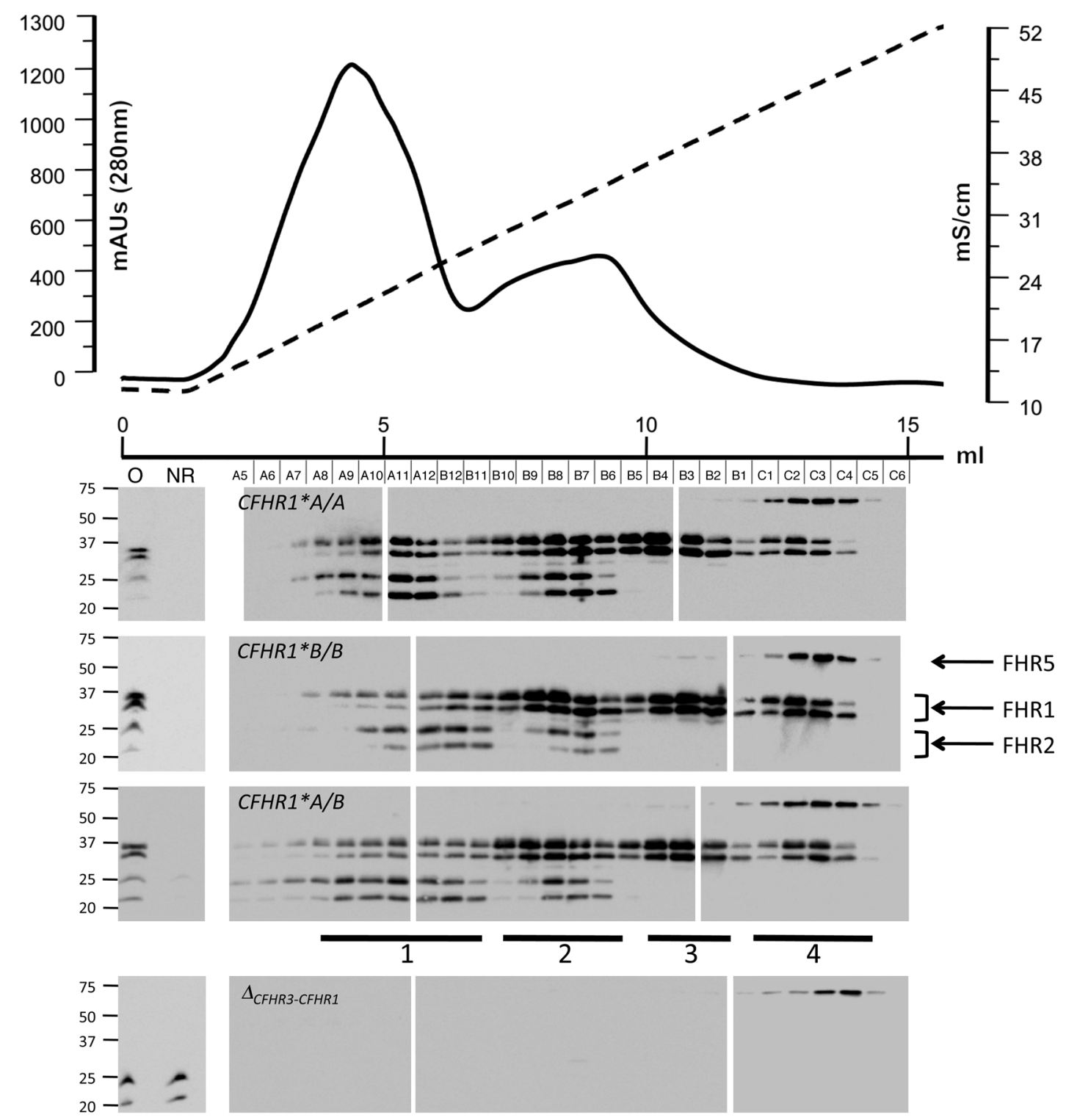

Figure 3

Heparin chromatography of FHR1, FHR2, and FHR5 proteins. Elution profiles of FHR1, FHR2, and FHR5 from individuals of the 3 CFHR1*A/B genotypes illustrated that FHR1 eluted in 4 distinct peaks at different $\mathrm{NaCl}$ concentrations. Top: Representative case of how plasma proteins retained in the heparin column were eluted with the $\mathrm{NaCl}$ gradient. Bottom: Western blots used the MBC125 mAb to determine the elution position of the different FHRs. Peaks 1-4 are indicated. FHR1 coeluted with FHR2 in the first 2 peaks: in peak 1, FHR1 and FHR2 showed equimolar quantities (FHR1-FHR2); in peak 2, FHR1 was approximately 3 times more abundant than FHR2 (FHR1 3 -FHR2). In peak 3, FHR1 eluted alone, and in peak 4, FHR1 and FHR5 coeluted at approximately equimolar quantities (FHR1-FHR5). FHRs were identified simultaneously by Western blot using MBC125 mAb, which recognizes an epitope common to the 3 FHRs. Protein bands corresponding to FHR1, FHR2, and FHR5 are indicated at right. Also shown is Western blot of the elution profile corresponding to an individual homozygote for the $\Delta_{C F H R 3-C F H R 1}$ allele, illustrating that FHR2 was not retained in the column in the absence of FHR1. Samples from the elution profiles were run in 3 separate gels. O, original; NR, nonretained.

$\mathrm{mM} \mathrm{NaCl}$ gradient. Although FHR1 was fully retained on the heparin column, this chromatographic fractionation did not result in a single elution peak containing FHR1; instead, FHR1 eluted in 4 distinct peaks at different $\mathrm{NaCl}$ concentrations. Immunodetection of FHR2 and FHR5 in the eluted fractions using the mouse $\mathrm{mAb}$ MBC125 (which recognizes an epitope common to FHR1, FHR2, and FHR5) showed that these 2 proteins, which were also fully retained in the heparin column, coeluted with FHR1 in the $\mathrm{NaCl}$ gradient (Figure 3). FHR2 coeluted with FHR1 in 2 distinct peaks: in peak 1, FHR1 and FHR2 appeared equimolar (FHR1-FHR2), whereas in peak 2, FHR1 was approximately 3 times more abundant than FHR2 (FHR13-FHR2). In peak 3, FHR1 eluted alone, and in peak 4, both FHR1 and FHR5 were present (FHR1-FHR5) (Figure 3). Identical results were obtained with FH-depleted plasma from individuals with each of the $3 C F H R 1{ }^{*} A / B$ genotypes (Figure 3 and ref. 17), which indicates that the peculiar FHR1 
A
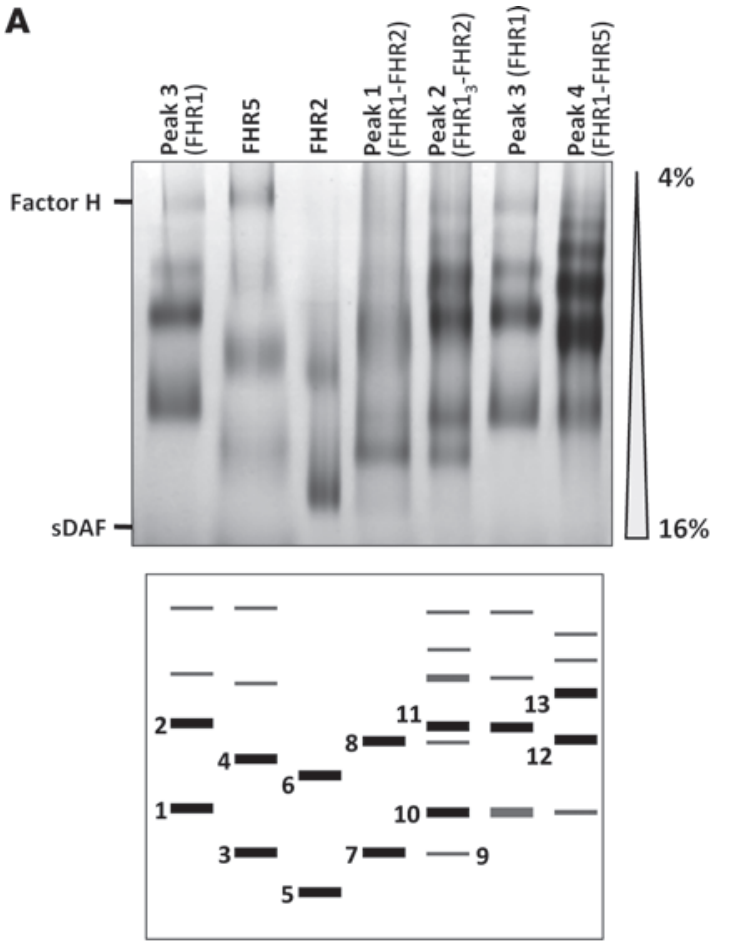

B

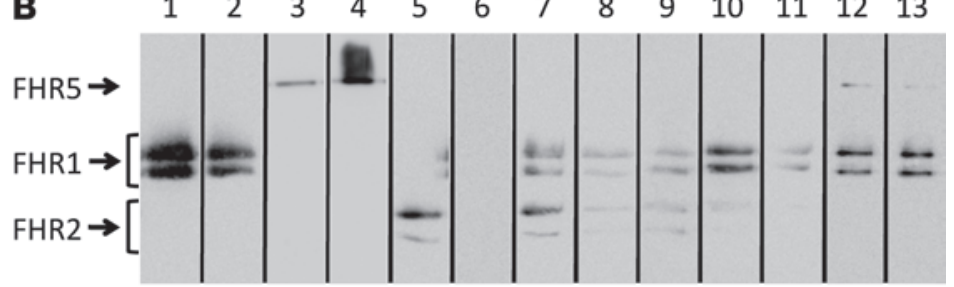

C

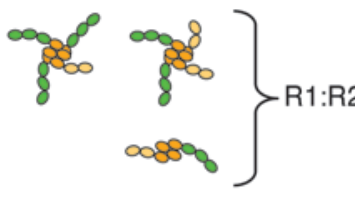

00800

R2
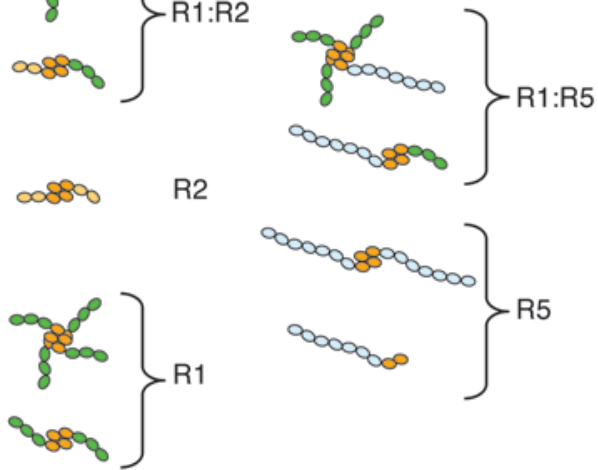

\section{Figure 4}

FHR1, FHR2, and FHR5 assemble into homo- and hetero-oligomers. (A) Purified FHRs corresponding to the 4 FHR1-containing peaks from heparin chromatography, and FHR2 and FHR5 proteins purified from a FHR1-deficient individual, were analyzed in 4\%-16\% polyacrylamide native gels. SDAF (4 SCRs) and FH (20 SCRs) were used as molecular weight markers. Gels were silver stained. Protein complexes were obtained for each sample (bottom). (B) FHR composition of the numbered protein complexes in A was analyzed by SDS-PAGE. Comparison of the relative mobility of the bands obtained for each sample, together with the Western blot analysis, demonstrated the presence of different homo- and hetero-oligomeric complexes. For example, lanes 1, 4, and 5 were interpreted as FHR1, FHR5, and FHR2 homodimers, respectively. A faint and diffuse band (lane 3) running below the band of the FHR1 dimer could correspond to a FHR5 monomer. Lane 6 did not contain FHR2 (likely a protein contamination). Therefore, there was only 1 protein complex in plasma containing FHR2 (likely a dimer). Lane 7 was a FHR1-FHR2 heterodimer: it contained both FHR1 and FHR2 and presented mobility intermediate between the FHR2 and FHR1 homodimers. Lanes 12 and 13 corresponded to different FHR1-FHR5 hetero-oligomers. Lanes were run on the same gel but were noncontiguous (black lines). (C) Putative structure of these complexes, based on structural data demonstrating that the first $2 \mathrm{~N}$-terminal SCRs (orange) of these proteins formed dimers in a head-to-tail orientation (13).

elution pattern was unrelated to the CFHR1 common polymorphism. Since no evidence of FHR1 posttranslational modifications was obtained to justify this complex FHR1 elution pattern, we concluded that it was a consequence of interaction of FHR1 with other FHRs. Moreover, because the analysis of FH-depleted plasma from individuals lacking FHR1 demonstrated that in the absence of FHR1, FHR2 was not retained in the heparin column (Figure 3), we postulated that FHR1 interacts with FHR2 and FHR5. Using this method, we could not see any evidence of an interaction between FHR2 and FHR5: we could not detect retained FHR2 when FHR1 was absent, and we did not observe coelution of FHR2 with FHR5 when FHR1 was present.

To determine whether FHR1 also interacts with FH, FHR3, and FHR4A/B, a sample of EDTA plasma from a $C F H R 1^{*} A / B$ heterozygote was passed through a MBC125 affinity column, and the presence of $\mathrm{FH}$ and all FHRs was analyzed in the flow-through and retained fractions by Western blot using a panel of specific Abs. Since FH, FHR3, and FHR4A/B proteins were not retained, we concluded that they do not interact with FHR1, FHR2, and FHR5, which were retained on the column (Supplemental Figure 4).

These experiments also showed that, when eluted from the MBC125 column at $\mathrm{pH} 2.5$ and renatured in PBS, FHR1, FHR2, and FHR5 demonstrated elution profiles from heparin columns identical to those obtained using FH-depleted plasma (Supplemental Figure 5). These data suggest either that the interactions between FHR1 and FHR2 (or FHR5) are very strong and are maintained through acid elution, or that formation of the different FHR complexes is a reversible process. In practical terms, this observation facilitated the development of a simple 2-step protocol for the purification of the different FHR1 complexes: affinity chromatographic purification from whole EDTA plasma using MBC125 mAb, and subsequent fractionation of the FHR1 complexes by heparin chromatography.

FHR1 assembles into bomo- and hetero-oligomeric complexes. Purified FHRs corresponding to the heparin chromatography elution peaks 1-4 (Figure 3), as well as FHR2 and FHR5 purified from the plasma of FHR1-deficient individuals, were analyzed by gel filtration using a Superdex 200 PC 3.2/30 column previously calibrated using 3 SCR-containing proteins of different sizes: FH (20 SCRs), C4bpa $\triangle \mathrm{CT}$ (8 SCRs), and soluble DAF (sDAF; 4 SCRs). $350 \mathrm{mM}$ $\mathrm{NaCl}$ was used in these experiments to avoid interaction of the FHRs with the carbohydrate components in the column matrix. Interestingly, the different purified FHRs eluted as a single peak with an apparent size that was consistent with the size of a FHR 


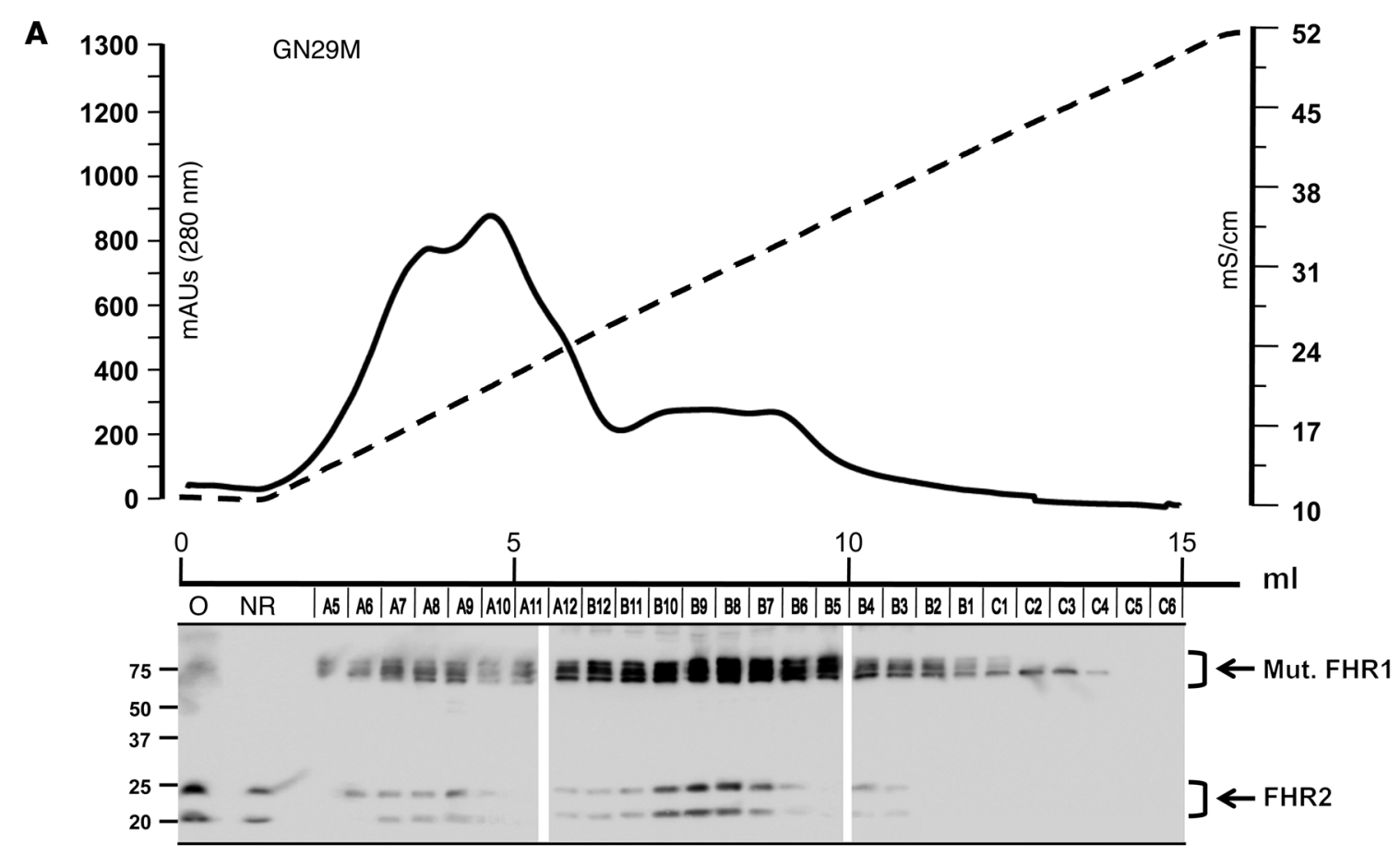

B

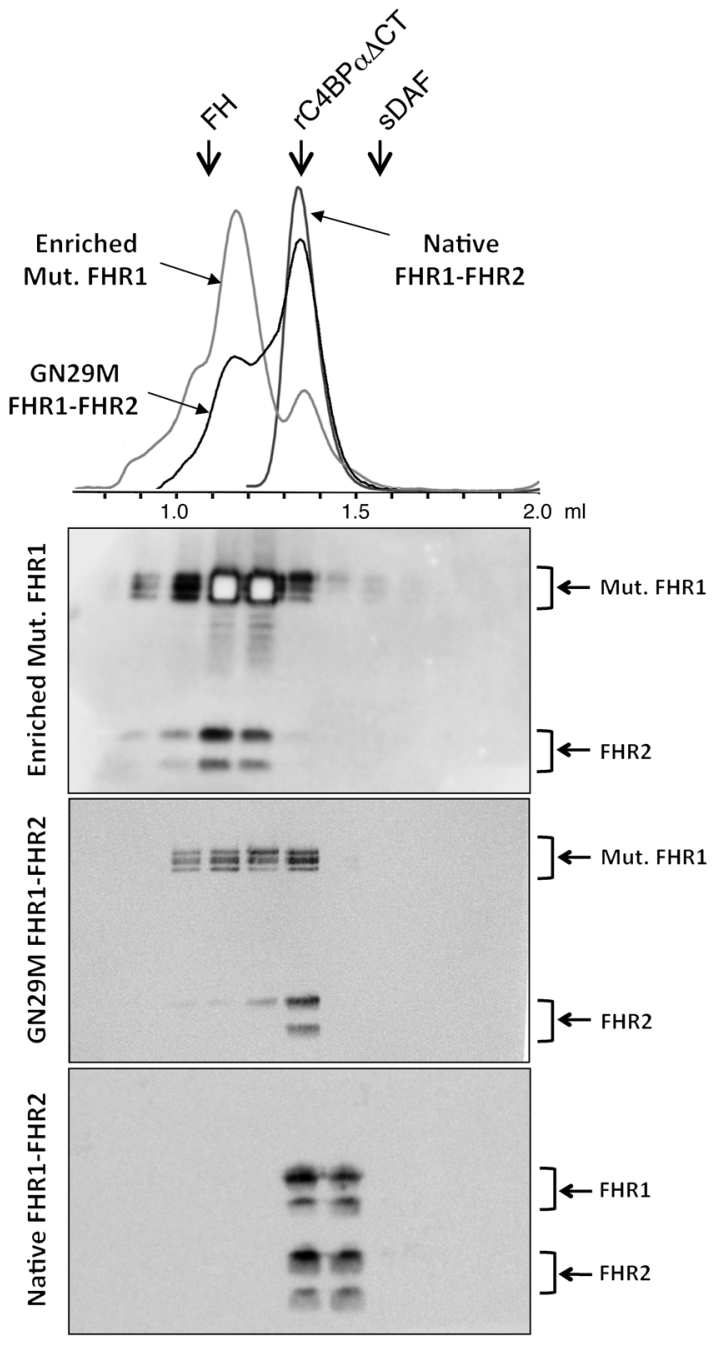

\section{Figure 5}

Mutant FHR1 protein shows an abnormal heparin chromatography elution profile and assembles into high-molecular weight multimers. (A) Elution profiles of the FHR1, FHR2, and FHR5 proteins from GN29M illustrated that the mutant FHR1 coeluted with FHR2 in a major peak extending through most of the $\mathrm{NaCl}$ gradient and a minor peak at low $\mathrm{NaCl}$ concentration. Heparin fractions were characterized by Western blot using MBC125. Samples from the elution profiles were run in 3 separate gels. (B) EDTA plasma from GN29M was passed through the MBC125 affinity column. The retained FHRs were eluted in $100 \mathrm{mM}$ glycine, $\mathrm{pH}$ 2.5; dialyzed against PBS; and analyzed by size-exclusion Superdex 200 PC 3.2/30 chromatography in $20 \mathrm{mM}$ Tris- $\mathrm{HCl}(\mathrm{pH} \mathrm{7.5)}$ and $350 \mathrm{mM} \mathrm{NaCl}$ (GN29M FHR1-FHR2). Purified proteins corresponding to the FHRs heparin chromatography elution peak 1 from a normal individual (Native FHR1-FHR2; corresponding to FHR1-FHR2 dimers) is also shown for size comparison. Also included is a preparation enriched of the high-molecular size species of the mutant FHR1 protein (Enriched Mut. FHR1). Proteins in the gel filtration fractions were characterized by Western blot and are shown below. 

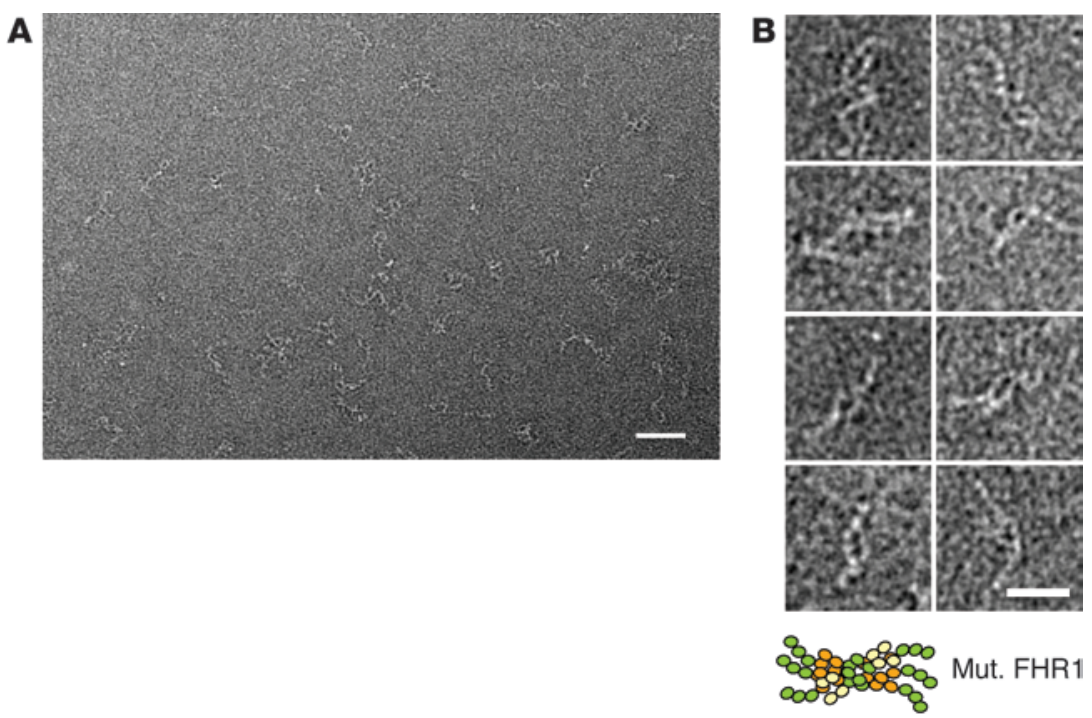

\section{Figure 6}

EM analyses of the mutant FHR1 high-molecular weight forms. (A) Typical field for a diluted sample of a negative-staining EM analysis of the purified mutant FHR1 enriched in high-molecular weight oligomers. Isolated complexes were detected as filaments of white density on the background of the micrograph. Scale bar: $50 \mathrm{~nm}$. (B) Gallery of selected images for single complexes, illustrating that each was composed of at least 2 elongated and flexible chains, presumably mutant FHR1 monomers. The length of individual chains was measured (about $30 \mathrm{~nm}$ ) and found to be in agreement with the estimated length of an elongated mutant FHR1 molecule composed of 9 SCR domains in tandem (approximately $3 \mathrm{~nm}$ per SCR domain). Scale bar: $25 \mathrm{~nm}$. dimer (Supplemental Figure 6), suggestive of the formation of homo- and heterodimers. The exception was FHRs in peak 4 (Figure 3), which eluted as 2 overlapping peaks. Evidence of FHR1FHR2 heterodimers was also obtained in immunoprecipitation experiments using $\mathrm{mAb}$ MBI-18, which specifically recognizes FHR2 (Supplemental Figure 7).

Purified proteins corresponding to the FHR heparin chromatography elution peaks $1-4$ were also analyzed in $4 \%-16 \%$ blue nativePAGE (BN-PAGE), which provided formal proof of the existence of homo- and hetero-oligomers of the FHRs (Figure 4). FH and sDAF were included in these native gels as molecular weight markers, and the FHRs were visualized by silver staining and Western blot analysis (Figure 4A). Bands from the native gels corresponding to the different complexes were excised and further analyzed in $12 \%$ SDS-PAGE gels (Figure 4B). As a whole, these experiments were consistent with the existence of homo- and heterodimers of the different FHRs. Notably, in samples containing FHR1, they also identified higher-order oligomers, which we interpreted as tetramers. For example, when purified FHRs included in peak 1 (fractions A11 to B11; Supplemental Figure 5) were run in the native gels, 2 FHR complexes were obtained (Figure 4A), both of which contained FHR1 and FHR2 (Figure 4B, lanes 7 and 8). Interestingly, the apparent sizes of the FHR oligomers containing FHR1 and FHR2 were intermediate between those obtained from peaks containing only FHR 1 or only FHR2, consistent with the size of the FHR1-FHR2 hetero-oligomers (Figure 4).

A tetrameric structure could explain the differences between peak 1 and peak 2 in the FHR heparin chromatography elution profiles (Figure 3). In fact, our native gels showed that peak 2 contained both FHR1-FHR1 homodimers and FHR1-FHR2 heterodimers, which suggests that the FHRs within this peak assemble into a tetramer formed by a FHR1-FHR1 homodimer and a FHR1-FHR2 heterodimer. In contrast, peak 1 only contained FHR1-FHR2 heterodimers, which suggests that it corresponds to a tetramer resulting from the interaction of 2 FHR1-FHR2 dimers. In agreement with this interpretation, when the purified proteins in peak 2 were denatured in $1 \mathrm{M} \mathrm{NaCl}$, dialyzed, and rechromatographed in the heparin column, they again displayed peaks 1-3 (Supplemental Figure 8).
Because we found only interactions among FHR1, FHR2, and FHR5, and these FHRs have almost identical N-terminal regions distinct from other FHRs, this region is the most likely candidate to mediate FHR oligomerization. It is unclear why FHR2 and FHR5 only formed homo-oligomers in the absence of FHR1.

The FHR1 mutant protein associated with C3G in the GN29 pedigree assembles into unusually large multimeric complexes. Consistent with the idea that the N-terminal region of FHR1 mediates homo- and hetero-oligomerization, analysis of the FHRs eluted from heparin chromatography from the FH-depleted plasma of GN29M showed an abnormal profile, with the mutant FHR1 coeluting with FHR2 through most of the $\mathrm{NaCl}$ gradient (Figure 5A). Moreover, gel filtration chromatographic analysis of the FHRs affinity purified from the EDTA plasma of GN29M using MBC125 revealed 2 major peaks containing the mutant FHR1 protein, one of them with a size considerably exceeding that of the normal FHRs analyzed under the same conditions (Figure 5B). We argued that these apparently large size structures were suitable for structural analysis by EM and set up experiments with purified native and mutant FHR1 complexes. Despite intensive effort, native FHR complexes could only be detected at high concentrations as an intersection of thin filaments corresponding to nearby molecules that were very close together. Unfortunately, these native FHR complexes were not visible upon dilution to the concentration required to separate individual molecules (data not shown). Similar behavior was observed for $\mathrm{FH}$, which we interpreted as a limitation of the EM method. Thin filaments like those formed by FH and FHR1 were at the size limit for observation using EM; thus, they were only detected when several molecules were very close together or part of a larger complex. In contrast, we were able to visualize protein complexes in a preparation enriched for the large-size mutant FHR1 complexes (Figure 6). Importantly, these structures were detected at concentrations when individual molecules were separate, which suggests that FHR1 mutant complexes are larger than native FHR1 proteins. Mutant FHR1 complexes appeared as multimeric, bundle-like structures, each containing several intersecting thin filaments. Interestingly, despite mutant FHR1 complexes displaying heterogeneous shapes with multiple arms in different orientations, their sizes were rather 

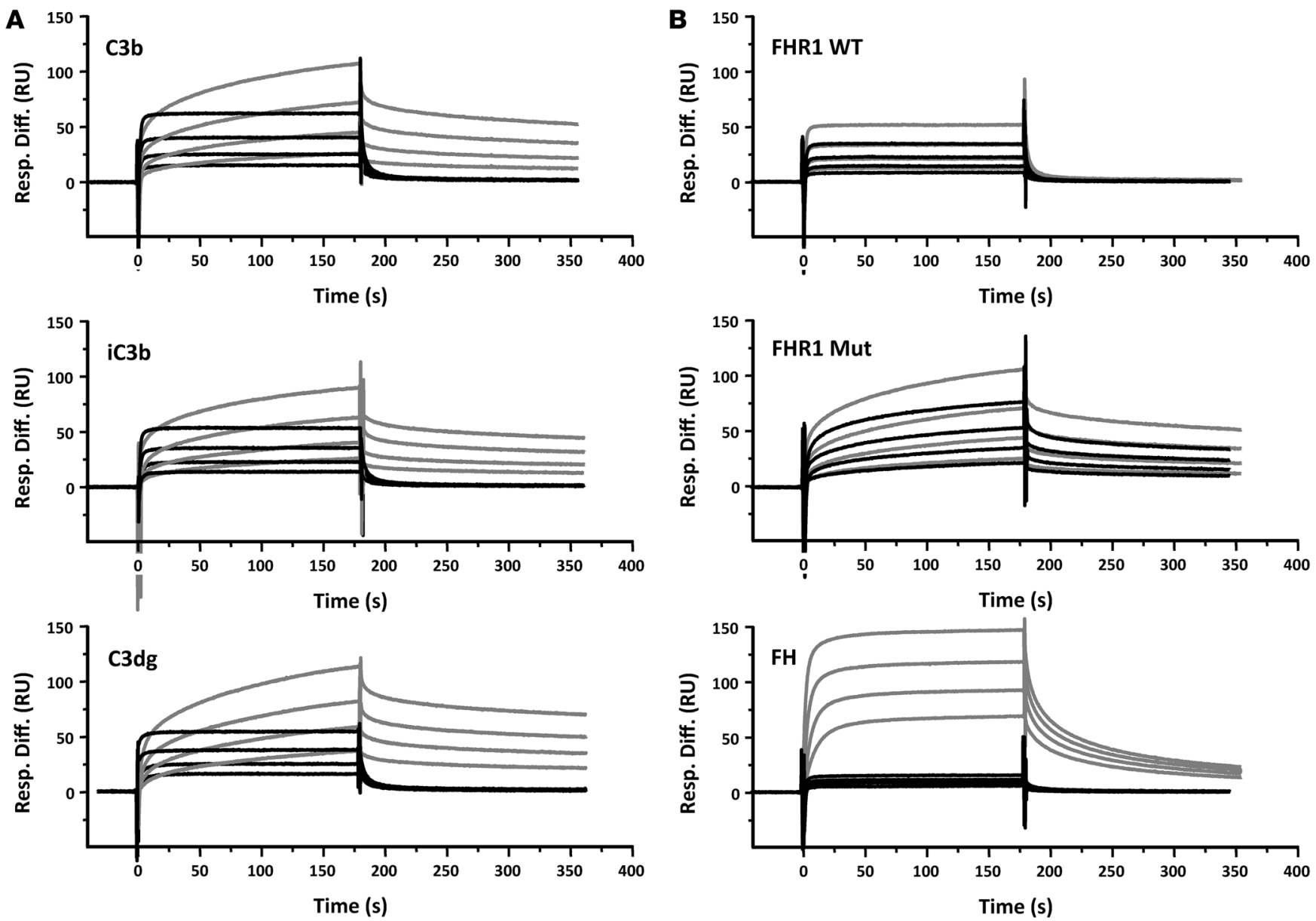

\section{Figure 7}

Mutant FHR1 shows increased binding to surface-bound C3b, iC3b, and C3dg. (A) SPR analysis of native (black lines) and mutant (gray lines) FHR1 binding to C3b, iC3b, and C3dg. C3b (150 RU) was amine-coupled to a CM5 Biacore chip and used as a nidus for convertase formation. Further C3b (1,240 RU) was deposited on the chip surface by flowing FB and FD to form C3bBb, followed by C3 as convertase substrate. The surface $\mathrm{C} 3 \mathrm{~b}$ was converted to iC3b by incubating with $\mathrm{FH}$ and FI until the surface no longer supported convertase formation. Conversion to $\mathrm{C} 3 \mathrm{dg}$ was achieved by incubating the iC $3 b$ surface with soluble CR1 and FI until no further C3c was released. 1:2 serial dilutions from $28 \mu \mathrm{g} / \mathrm{ml}$ native and mutant FHR1 were flowed across the surface for 3 minutes, then allowed to dissociate for 3 minutes prior to regenerating the surface. Avidity effects affecting dissociation of mutant FHR1 are clear. (B) Comparative binding of native and mutant FHR1 and FH to C3b and iC3b. Serial dilutions of native FHR1 (WT; $28 \mu \mathrm{g} / \mathrm{ml}$ ), mutant FHR1 (Mut; $28 \mu \mathrm{g} / \mathrm{ml}$ ), and FH (bottom; $26 \mu \mathrm{g} / \mathrm{ml}$ ) were flowed across the C3b surface (gray lines) as described in $\mathbf{A}$. The surface $\mathrm{C} 3 \mathrm{~b}$ was converted to iC3b by incubating with $\mathrm{FH}$ and $\mathrm{Fl}$ until the surface no longer supported convertase formation. Native and mutant FHR1 as well as FH were flowed across iC3b (black lines) at identical concentrations as in $\mathbf{A}$. Binding of native or mutant FHR1 was little affected by conversion of $\mathrm{C} 3 \mathrm{~b}$ to $\mathrm{iC} 3 \mathrm{~b}$, whereas binding of $\mathrm{FH}$ was almost eliminated.

homogeneous, which indicates that the dimensions of the complexes found were within a discrete range, and very large aggregates were not detected. As a whole, the EM data support that mutant FHR1 is assembled into a larger multimeric complex.

GN29 mutant FHR1 shows increased interaction with surface-bound $C 3 b, i C 3 b$, and $C 3 d g$. To explore the functional consequences of the effect of multimerization on the mutant FHR1, we performed surface plasmon resonance (Biacore) experiments. Because we could not demonstrate complement inhibitory capabilities for FHR1 in preliminary analyses (Supplemental Figures 9-11), we therefore focused on the interaction of FHR1 with surface-bound C3 fragments. Our results showed that when C3b was immobilized on a CM5 Biacore chip through the thioester group, mutant FHR1 bound more efficiently to C $3 \mathrm{~b}$ than the native FHR1 protein. The results illustrated fast binding and dissociation kinet- ics for the native FHR1 protein, whereas binding of the mutant FHR1 showed much slower dissociation (Figure 7A). As expected, these results support the hypothesis that multimerization of FHR1 in the mutant protein increases avidity for its ligands, leading to much enhanced binding to $\mathrm{C} 3 \mathrm{~b}$ compared with native FHR1. Similar results were obtained with iC3b and C3dg. Binding of native and mutant FHR1 was comparable to that of $\mathrm{FH}$ on the same surfaces: FH bound well to the C3b surface, illustrating marked avidity effects as would be expected on the "clustered C $3 b$ " surface. Conversion to iC $3 b$ almost eliminated binding of $\mathrm{FH}$, whereas native and mutant FHR1 bound almost as well to $\mathrm{iC} 3 \mathrm{~b}$ as to $\mathrm{C} 3 \mathrm{~b}$ (Figure $7 \mathrm{~B}$ ).

GN29 mutant FHR1 shows enhanced competition with FH. As a consequence of binding more efficiently to its ligands, the mutant FHR1 protein may compete better with FH. To investigate this 


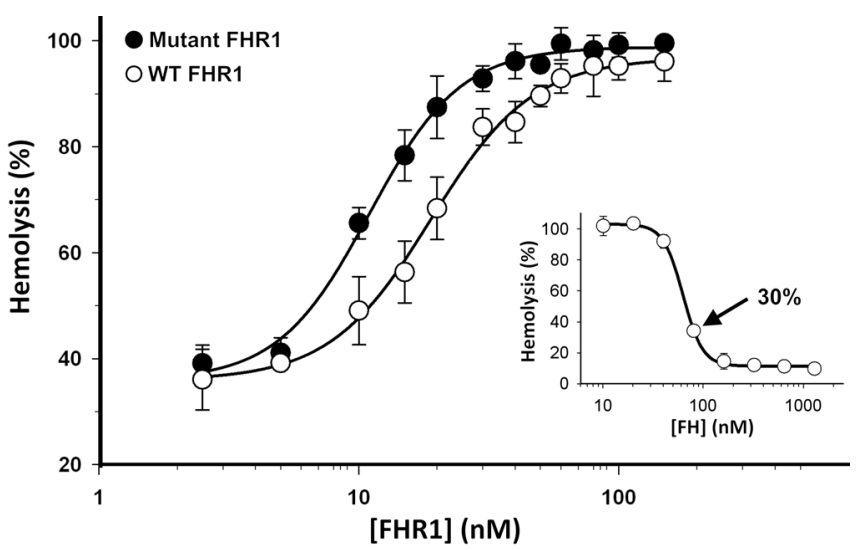

possibility, we tested native and mutant FHR1 in a variation of the FH-dependent hemolytic assay previously developed in our laboratory (12). In this modified assay, a human serum sample was incubated with guinea pig erythrocytes (instead of the sheep erythrocytes used in the original assay) in the presence of $5 \mathrm{mM}$ $\mathrm{MgCl}_{2}$ and $8 \mathrm{mM}$ EGTA. Guinea pig erythrocytes were efficiently lysed in this assay, but lysis could be prevented by the addition of extra FH (Figure 8, inset). Importantly, FHR1 competed with FH in a dose-dependent manner, influencing control of the AP on the surface of the guinea pig erythrocytes. As shown in Figure 8, the mutant FHR1 protein competed with $\mathrm{FH}$ more efficiently than did native FHR1. These data indicate that multimerization as a consequence of duplication of the $\mathrm{N}$-terminal region enhances competition with $\mathrm{FH}$ for certain surfaces, resulting in increased activation of the complement AP.

\section{Discussion}

We report here the identification of a mutant FHR1 protein (carrying a duplication of the N-terminal region conserved in FHR1, FHR2, and FHR5) in a Spanish pedigree with multiple C3G-affected individuals. This finding made us aware of an important genotype-phenotype correlation, because similar duplications in FHR5 and FHR2 have been previously associated with other familial cases of C3G. To understand the significance of this peculiar genotypephenotype association, we performed a series of biochemical and structural analyses with the native and mutant FHR1 proteins that unraveled an unexpected structural organization of FHR1, FHR2, and FHR5 in human plasma. We showed that FHR1 assembled into homo- and hetero-oligomers with FHR2 and FHR5, but did not interact with other related proteins, like FHR3, FHR4A/B, FH, or FHL1, which lack the 2 conserved N-terminal SCRs. We also showed that the mutant FHR1 with the duplicated N-terminus generated abnormally large multimers, which appeared as a bundle of fibers as visualized with EM (Figure 6). Together, these data suggest that the duplicated $\mathrm{N}$-terminal region of FHR1 includes an oligomerization domain and point to the $2 \mathrm{~N}$-terminal SCRs conserved in FHR1, FHR2, and FHR5 as the most likely candidate domain for this activity. This conclusion is also supported by recent structural data demonstrating that the first $2 \mathrm{~N}$-terminal domains of these proteins form dimers (13). The location of the oligomerization domain at the $\mathrm{N}$-terminus revealed that the FHRs are bifunctional molecules and explains why the FHRs in these complexes retain their capacity to interact with $\mathrm{C} 3 \mathrm{~b}$ and carbohydrates, activities located in the C-terminal region of FHRs $(1,16,17)$.

\section{Figure 8}

Mutant FHR1 shows enhanced competition with $\mathrm{FH}$ in a FH-dependent hemolytic assay. Inset shows the preliminary experiment to determine the amount of $\mathrm{FH}$ to give $30 \%$ lysis in the guinea pig hemolytic assay. Main plot shows the competition assay between native FHR1 (open circles) or mutant FHR1 (filled circles) and FH amount. Percent hemolysis was determined as mean \pm SD of 3 independent experiments. The $\mathrm{EC}_{50}$ values $-18.97 \mathrm{nM}$ for native FHR1 and $10.97 \mathrm{nM}$ for mutant FHR1 — were significantly different $(P=0,001,2$-tailed $t$ test).

The structural organization of the FHRs in plasma was complex, but remarkably constant among different individuals, as illustrated by the elution profiles from heparin columns (Figure 3). These experiments also showed that FHR1, the most abundant FHR in plasma, played a central role in this structural organization. We identified 4 different types of complexes that incorporated FHR1 and, using native gels, provided evidence of the existence of homo- and heterodimers, as well as higher-order oligomeric structures (interpreted here as tetramers), resulting from the different combinations of FHR1-FHR1, FHR1-FHR2, and FHR1-FHR5 dimers. The FHR composition of the different oligomers influenced their elution position from the heparin column, indicative of different carbohydrate binding characteristics for each of these FHR complexes.

These findings have very important functional implications. By assembling into homo- and hetero-oligomeric complexes, FHR1, FHR2, and FHR5 likely increase their avidity for specific surfaces, which supports the concept that these FHRs influence complement activation and regulation by efficiently competing with $\mathrm{FH}$ for binding to those surfaces. This is particularly relevant in light of the finding that FHRs, but not FH, bound $\mathrm{iC} 3 \mathrm{~b}$ and $\mathrm{C} 3 \mathrm{dg}$ (Figure 7B), giving these oligomeric FHR structures a considerable advantage for competing with $\mathrm{FH}$ on surfaces where $\mathrm{iC} 3 \mathrm{~b}$ and $\mathrm{C} 3 \mathrm{dg}$ are present as a consequence of complement activation. In this respect, it is important to indicate that the FHR1 oligomers did not show cofactor activity for the FI-mediated inactivation of iC3b, nor did they inhibit the proteolysis of iC3b by CR1 and FI (Supplemental Figure 12). In addition, the generation of a combinatorial repertoire of different FHRs may improve surface recognition, fine-tuning the competition with $\mathrm{FH}$.

Notably, we here describe 2 situations in which this precise FHR structural organization is disrupted, with very different outcomes. The first is the case of individuals homozygous for the $\Delta_{\text {CFHR3-CFHRI }}$ allele, who lack these FHR1-containing complexes; the second is the case of the mutant FHR1 protein. Interestingly, while the former confers protection to a number of chronic diseases characterized by complement dysregulation, the anomalous FHR1 oligomerization caused by the duplication of the oligomerization domain had pathogenic consequences, as illustrated by the finding that it was associated with $\mathrm{C} 3 \mathrm{G}$.

The pathogenic consequences of the multimerization of mutant FHR1 are likely related to increased avidity for the FHR1 ligands, which enhances competition with $\mathrm{FH}$ and impairs complement regulation on surfaces. We showed that this was the case for the 


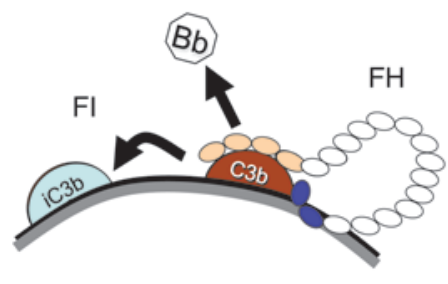

Normal

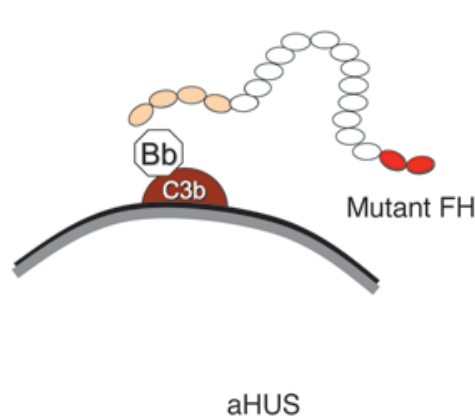

aHUS

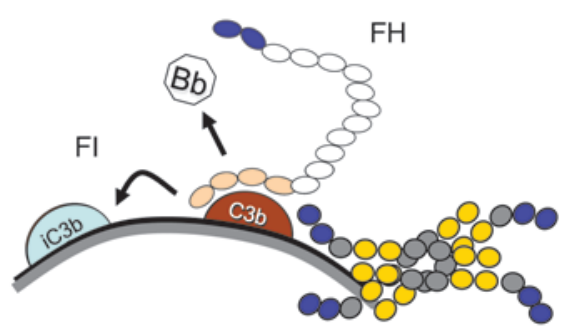

Mutant FHR1

C3G

\section{Figure 9}

Proposed model for a novel pathogenic mechanism in C3G. FH is an elongated glycoprotein of $155 \mathrm{kDa}$ composed of $20 \mathrm{SCRs}$ (small circles). FH presents $\mathrm{C} 3 \mathrm{~b}$ binding sites at each end of the molecule. The $\mathrm{N}$-terminal C3b binding site mediates the accelerated decay of the AP C3 convertase $(\mathrm{C} 3 \mathrm{bBb})$ and the cofactor activity for the $\mathrm{Fl}$-dependent proteolytic inactivation of $\mathrm{C} 3 \mathrm{~b}$. The $\mathrm{C}$-terminal region binds both $\mathrm{C} 3 \mathrm{~b}$ and polyanions normally present in the cell surfaces (e.g., sialic acid, heparan sulfates, and glycosaminoglycans). This region is essential for the complement regulatory activity of $\mathrm{FH}$ on surfaces and to discriminate between self and pathogens, which normally lack these polyanions on their surfaces. Extensive experimental data generated during the last 10 years has provided conclusive evidence that mutations disrupting the functional activity of the C-terminal region, like those associated with aHUS, decrease the avidity of $\mathrm{FH}$ for cell surfaces and impair complement regulation (34-36). The data reported here suggest similarities between the established model for the aHUS-associated FH mutations and the FHR1 mutant described here. We therefore propose that multimerization of the FHRs as a consequence of duplication of the oligomerization domain in mutant FHR1, FHR2, or FHR5 proteins increases binding to surface-bound C3b, $\mathrm{iC} 3 \mathrm{~b}, \mathrm{C} 3 \mathrm{dg}$, and carbohydrates, resulting in enhanced competition with $\mathrm{FH}$ that decreases its complement regulatory capacity and causes different degrees of cell surface complement dysregulation.

interaction with surface-bound C3b (Figure 7) and provided evidence using a hemolytic assay that the mutant FHR1 competed more efficiently than did native FHR1 to reduce the protection against complement-mediated lysis conferred by FH (Figure 8). Thus, excessive competition between the mutant FHR1 and FH represents a novel way to cause complement dysregulation and is likely the pathogenic mechanism in our C3G pedigree (Figure 9).

The way that mutant FHR1 causes complement dysregulation resembles the effects of C-terminal $\mathrm{FH}$ mutations that are associated with aHUS. In both cases, the consequence is decreased interaction between $\mathrm{FH}$ and the surface where complement activation needs to be regulated. Why, then, does the excessive competition of FH by the mutant FHR1 not result in aHUS? We believe that although the FH mutations associated with aHUS likely impair binding of FH to most self-surfaces, FHR1 competes the binding of FH to only a subset of those surfaces, and this subset does not include the endothelial cell surface. Evidence in support of this proposal includes a sheep hemolytic assay in which the binding of FH cannot be competed by FHR1 and inability of the hybrid FH::FHR1 protein associated with aHUS (in which the $\mathrm{C}$-terminus of $\mathrm{FH}$ has been replaced by the $\mathrm{C}$-terminus of FHR1) to protect cell surfaces from complement lysis $(18-20)$. It is presently unclear on which surfaces FH would be primarily competed by the mutant FHR1 protein. As presented here, FHR1, FHR2, and FHR5 are deregulators of complement activation that, by competing $\mathrm{FH}$, enhance AP activation on certain surfaces. One possibility that deserves further investigation is that the FHRs originated through evolution to prevent binding of $\mathrm{FH}$ to certain pathogens and that their microbial ligands or cross-reacting physiological substances deposited in the GBM are the sites of competition between FHR1 and FH. Our proposal assumes that there must be low-level C3 activation along the GBM (spontaneous, following a trigger, or both) that is accelerated in the presence of these mutant proteins. In this respect, it is perhaps not coincidental that the episodes of disease exacerbation in our patients and in other $\mathrm{C} 3 \mathrm{G}$ patients carrying similar mutant FHRs frequently occur concurrent with infection and fever episodes.

Our data, and previous genetic association data indicating that deletion of the CFHR1 and CFHR3 genes $\left(\Delta_{\text {CFHR3-CFHR1 }}\right)$ is a lower risk for factor for AMD (9) and IgA nephropathy (10), suggest that the relative amounts of $\mathrm{FH}$ and FHR1 may influence disease by modulating complement regulation on certain surfaces. In the family studied herein, the mother, but not her affected son, carried the $\Delta_{\text {CFHR3-CFHR1 }}$ allele and therefore lacked native FHR1. Whether this differential expression of native FHR1 relates to the small variations in their disease phenotypes, including the small differences in the location of the dense deposits observed in the kidney biopsies of the mother and son, requires further investigation.

In summary, our identification and functional characterization of a mutant FHR1 protein associated with $\mathrm{C} 3 \mathrm{G}$ provided insights into the biological role of FHRs and has improved our understanding of the ways in which mutant FHRs cause pathology. Previous studies have shown that the control of complement activation is impaired in C3G patients and that dysregulation of the complement system may result either from loss of activity of the complement regulatory protein $\mathrm{FH}$ (21) or from gain-offunction mutations in $\mathrm{C} 3$ that confer to the AP convertase resistance to FH inactivation (22). Those studies also indicated that complement dysregulation in prototypical DDD occurs primarily in the fluid phase and results in the complete consumption of $\mathrm{C} 3$. Consistent with the heterogeneity among C3G patients, we show here that complement dysregulation on surfaces may also occur in other forms of $\mathrm{C} 3 \mathrm{G}$ and describe a novel pathogenic mechanism involving an abnormal multimeric FHR1 protein that likely causes surface dysregulation. The finding that some C3G patients have surface and not fluid-phase complement dysregulation has important therapeutic implications. We speculate that it could 
explain why treatment of $\mathrm{C} 3 \mathrm{G}$ patients with eculizumab, a humanized mAb blocking C5 activation, led to an improvement in renal parameters in only approximately half of patients (23-27). The pathophysiological heterogeneity of $\mathrm{C} 3 \mathrm{G}$ means that the appropriate complement-inhibiting strategy will be determined by the underlying mechanism of complement dysregulation.

\section{Methods}

Patients. GN29 is a 12-year-old boy who, coincident with an episode of fever associated with an upper respiratory infection and purulent conjunctivitis by Haemophilus influenzae, presented with macroscopic hematuria at 15 months of age. 1 month earlier, he had a similar episode that resolved spontaneously in 3 days. At that time, moderate fever was also present, as well as the macroscopic hematuria and subnephrotic-range proteinuria. Blood pressure and renal function were normal. Plasma C3 levels were slightly below the normal range. A third episode, similar to the previous ones, occurred at age 20 months, precipitating a nephrology review. Despite preservation of renal function and normotension, he had persistent microhematuria of variable intensity that becomes macroscopic during intercurrent fever. Proteinuria remained in the subnephrotic range with no peripheral edema. However, in 2009, he developed edema and nephrotic-range proteinuria that was steroid responsive, coincident with a drop in plasma $\mathrm{C} 3$ levels (Figure 2B). Current medication is angiotensin converting enzyme inhibitor (ACEI) therapy, and proteinuria is now in the subnephrotic range. Notably, his $\mathrm{C} 3$ levels in plasma have always been below the normal range. A renal biopsy performed in 2008 revealed glomerular hypertrophy with diffuse capillary and mesangial thickening and hypercellularity (Figure 1A). The GBM was thick due to the linear accumulation of PAS-positive material that was also found locally in the mesangium (Figure 1, B and C). Immunofluorescence studies revealed very intense granular C3 staining along thickened capillary loops (Figure 1D) and within the mesangium, where focal IgM staining was also present (Figure 1E). Staining for IgG was negative (Figure 1F). Transmission EM demonstrated the presence of osmiophilic deposits in the GBM and mesangium (Figure 1G).

GN29M, mother of GN29, had microhematuria, trace proteinuria, arterial hypertension, and decreased plasma C3 levels in a routine screening in 1999 , prior to the in vitro fecundation during her first and only twin pregnancy. She delivered by Caesarean section because of preeclampsia. Since 2002, her hypertension has been controlled with ACEI treatment and diet. Her renal function is preserved $(0.79 \mathrm{mg} / \mathrm{dl}$ creatinine, corresponding to a creatinine clearance rate of $140 \mathrm{ml} / \mathrm{min}$ ). She maintains a persistent variable proteinuria (trace to $50 \mathrm{mg} / \mathrm{dl}$ ), moderate microhematuria (>50 erythrocytes/field), and chronic hypocomplementemia, with levels of plasma C3 persistently below the normal range. A renal biopsy performed in 2009 showed a C3G with intense C3 deposits similar to those observed in her son, negative IgG and IgM staining, and the presence of subendothelial and subepithelial GBM and mesangium osmiophilic deposits (Figure 1, H-O).

GN29GF, maternal grandfather of GN29, developed chronic renal insufficiency at 51 years of age. Renal biopsy diagnosis was membranoproliferative glomerulonephritis type II (now DDD). 6 years later, he presented with acute myocardial infarction and multivessel coronary artery disease. In 2001, he reached end-stage renal disease. He received a renal transplant, but died in 2007 from acute myocardial infarction.

Mutation screening and genotyping. Genomic DNA was obtained from peripheral blood mononuclear cells using Puregene Blood Core kit B (QIAGEN) according to manufacturer's instructions. Each exon of the $C F H$, $M C P, C F I, C 3$, and $C F B$ genes was amplified from genomic DNA using specific primers derived from the $5^{\prime}$ and $3^{\prime}$ intronic sequences, as described previously $(2,22,28-30)$. Automatic sequencing was performed in an ABI 3730 sequencer using a dye terminator cycle sequencing kit (Applied Biosystems).
MLPA analysis and bigh-density CGH arrays. Copy number variations and genomic rearrangements in the $C F H$-CFHR1-CFHR5 region were assessed by MLPA analysis and custom-designed high-density $8 \times 15 \mathrm{k}$ oligonucleotide CGH arrays spanning the RCA gene cluster region in human chromosome 1q32 (median resolution, 110 bp; AMADID 040193; Agilent Technologies). MLPA analysis was done with genomic DNA with the P236 A1 ARMD mix 1 (MRC-Holland). For the CGH $8 \times 15 \mathrm{k}$ microarrays, samples were hybridized with sex-matched normal human DNA (Promega Corp.) according to the manufacturer's protocols (31). Microarray data were extracted and visualized using Feature Extraction Software (version 10.7) and Genomic Workbench Standard Edition (version 7.0; both Agilent Corp). Copy number-altered regions were detected using ADM-2 (set as 5) statistic provided by DNA Analytics, with a minimum number of 5 consecutive probes. Genomic build hg19 was used for the experiment. All work involved in the hybridization of the CGH $8 \times 15 \mathrm{k}$ microarrays and data extraction was outsourced to Nimgenetics. Microarray data have been deposited in GEO (accession no. GPL16749).

Proteins and Abs. C3b was prepared as previously described from human EDTA plasma (32). sDAF was a gift from S. Lea (Oxford University, Oxford, United Kingdom). C4bp $\alpha \Delta C T$ was prepared as previously described (33). FH from human plasma was purified by immunoaffinity chromatography. Briefly, filtered plasma was loaded into an immunoaffinity column coupled to an in-house anti-human FH mouse mAb (mAb214). Bound protein was eluted at low $\mathrm{pH}$, and fractions containing the protein were pooled and loaded into a Superose-6 column equilibrated with $10 \mathrm{mM}$ HEPES (pH 7.6) and $100 \mathrm{mM} \mathrm{NaCl}$. MBC125 is a mouse IgG2a mAb raised against a recombinant protein containing the first $3 \mathrm{SCRs}$ of FHR1. This Ab recognizes an epitope present in FHR1, FHR2, and FHR5 and thus allows for simultaneous quantitative detection of the 3 proteins in Western blot analyses. Other Abs were in-house mouse mAb MBI-18 against FHR2 (gift from B.P. Morgan, Cardiff University School of Medicine, Cardiff, United Kingdom); in-house rabbit polyclonal $\mathrm{Ab}$ against FHR3 and FHR4 and rabbit polyclonal $\mathrm{Ab}$ against FHR4 (gifts from P.F. Zipfel and M. Jozsi, Hans Knöll Institute for Natural Products Research, Jena, Germany); and OX24, a mouse mAb to FH originally made by R. Sim (University of Oxford, Oxford, United Kingdom).

$B N-P A G E$. Samples were analyzed by BN-PAGE. Briefly, the different FHRs purified from the heparin columns were dialyzed overnight against $20 \mathrm{mM}$ HEPES, pH 7.0, and $150 \mathrm{mM} \mathrm{NaCl}$. Samples were mixed with loading buffer (50 mM BisTris, pH 7.2; $50 \mathrm{mM} \mathrm{NaCl;} 10 \%$ [v/v] glycerol; $0.001 \%$ $[\mathrm{v} / \mathrm{v}]$ Ponceau S; $0.25 \%[\mathrm{w} / \mathrm{v}]$ Coomassie G-250) and loaded on precast BNPAGE gels (Novex BisTris; Invitrogen). Electrophoresis was performed at $4^{\circ} \mathrm{C}$ according to the manufacturer's protocol, and proteins were identified by silver staining and Western blot. The composition of each band detected by BN-PAGE was analyzed by $12 \%$ SDS-PAGE followed by Western blot using the MBC125 mAb. Silver staining and Western blots were carried out according to standard protocols.

Biosensor analysis/binding affinity assays using SPR. All analyses were carried out on a Biacore T100 (GE Healthcare). A nidus of C3b (150 RU) was aminecoupled to a CM5 (carboxymethylated dextran) sensor chip as instructed by the manufacturer (NHS/EDC coupling kit; GE Healthcare). Further C3b was deposited via the internal $\mathrm{C} 3$ thioester group by forming convertase on amine-coupled C3b by flowing $50 \mu \mathrm{g} / \mathrm{ml} \mathrm{FB}$ and $1 \mu \mathrm{g} / \mathrm{ml}$ factor D (FD) using the same buffer supplemented with $1 \mathrm{mM} \mathrm{MgCl}_{2}$, followed by $\mathrm{C} 3$ as substrate for the convertase (34). 1,240 RU nascent C3b was covalently bound to the chip surface. Coupling in this manner results in "clustered" $\mathrm{C} 3 \mathrm{~b}$ on the surface as would be present on a target. To generate iC $3 \mathrm{~b}$, the C3b surface was treated with successive cycles of FH $(10 \mu \mathrm{g} / \mathrm{ml})$ and FI (10 $\mu \mathrm{g} / \mathrm{ml}$ ) until convertase could no longer be formed. To generate $\mathrm{C} 3 \mathrm{dg}$, the iC3b surface was treated with successive cycles of soluble CR1 $(50 \mu \mathrm{g} / \mathrm{ml}$; gift from T Cell Sciences) and FI (10 $\mu \mathrm{g} / \mathrm{ml})$ until no further loss of mass (C3c) was evident. To assess binding to C3b, iC $3 \mathrm{~b}$, or $\mathrm{C} 3 \mathrm{dg}$, samples were injected 
using the "High Performance" command, in HBS/P (10 mM HEPES, pH $7.4 ; 150 \mathrm{mM} \mathrm{NaCl} ; 0.01 \%$ surfactant-P20) flowed at $20 \mu \mathrm{l} / \mathrm{min}$ and analyzed at $25^{\circ} \mathrm{C}$. All data were double-referenced (data from control cell and blank injection subtracted). The chip surface was regenerated between injections using $10 \mathrm{mM}$ sodium acetate, $\mathrm{pH} 4.0$, and $1 \mathrm{M} \mathrm{NaCl}$. Data were evaluated using Biaevaluation software (version 4.1; GE Healthcare).

FH-dependent hemolytic assay. The capacity of FHR1 and mutant FHR1 to compete the activity of FH to control AP on cellular surfaces was assessed with a hemolytic assay using guinea pig erythrocytes. In brief, guinea pig erythrocytes (TCS Biosciences) in AP buffer ( $0.5 \%$ packed cell volume) consisting of veronal buffer saline $(2.5 \mathrm{mM}$ barbital; $1.5 \mathrm{mM}$ sodium barbital; $144 \mathrm{mM} \mathrm{NaCl}, \mathrm{pH} 7.4$ ) with $5 \mathrm{mM} \mathrm{MgCl}_{2}, 8 \mathrm{mM}$ EGTA, and $0.1 \%$ gelatin - were incubated with sufficient amounts of $10 \%$ serum in AP buffer and increasing amounts of $\mathrm{FH}$ for 1 hour at $37^{\circ} \mathrm{C}$. After centrifugation, supernatants were read at $414 \mathrm{~nm}$. Titration of FH before each experiment determined conditions for the competition assay. The minimal concentration of FH giving $30 \%$ lysis was mixed with different amounts of FHR1 proteins to measure their capacity to compete with FH (starting concentration, $150 \mathrm{nM}$ ). Serum without added $\mathrm{FH}$ was taken as $100 \%$ lysis, and erythrocytes diluted in AP buffer were used as blank for spontaneous lysis.

EM analyses. Mutant FHR1 preparations were subjected to a final purification step using size-exclusion Superdex 200 chromatography (GE Healthcare) in $20 \mathrm{mM}$ Tris- $\mathrm{HCl}, \mathrm{pH} 7.5$, and $350 \mathrm{mM} \mathrm{NaCl}$ before the preparation of EM grids. A selected fraction was adsorbed onto glow discharged carbon-coated grids and negatively stained with $2 \%(\mathrm{~m} / \mathrm{v})$ uranyl formate. Grids were visualized in a JEOL 1230 transmission electron microscope operated at $100 \mathrm{kV}$, and micrographs were recorded on a $4 \mathrm{k} \times$ $4 \mathrm{k}$ TVIPS CMOS detector at a nominal magnification of $\times 50,000$.

Statistics. Statistical analysis of the FH-dependent hemolytic assay data in Figure 8 was done by 2 -tailed $t$ test (independent samples). The analysis was performed using SPSS software (version 21; SPSS). Results are expressed as a mean of 3 independent experiments. A $P$ value of 0.05 or less was considered significant.

Study approval. The studies described herein received IRB approval (Comision de Bioetica, Consejo Superior de Investigaciones Científicas). Patients and their relatives gave their informed consent.

\section{Acknowledgments}

We acknowledge Susan Lea (University of Oxford, Oxford, United Kingdom), who, prior to making her findings public, showed us the crystal structure of the N-terminal region of FHR1, demonstrating that the protein domain including SCR1 and SCR2 mediates the dimerization of the FHR1, FHR2, and FHR5 proteins in a head-to-tail orientation. We also thank B. Paul Morgan for constant support, comments on the manuscript, and provision of $\mathrm{mAb}$ against FHR2. We are grateful to the patients and their relatives for their participation in this study. Work in this report was funded by the Spanish Ministerio de Economía y Competitividad (SAF2011-26583), the Fundación Renal Iñigo Alvarez de Toledo and the 7FP European Union project EURenOmics to S. Rodríguez de Córdoba; the "Ramón Areces" Foundation, the Spanish Ministerio de Economía y Competitividad (SAF2011-22988), and the "Red Temática de Investigación Cooperativa en Cáncer (RTICC)" (RD06/0020/1001) to O. Llorca; the Spanish "Ministerio de Economía y Competitividad" (PI0900268) to P. SánchezCorral; the MRC UK (G0701298) to C.L. Harris; and the Spanish "Ministerio de Economía y Competitividad" (PS09/00122) to M. López Trascasa. In addition, this work was supported by a grant from the Autonomous Region of Madrid (S2010/BMD-2316) to S. Rodríguez de Córdoba, O. Llorca, M. López Trascasa, and P. Sánchez-Corral. M.C. Pickering is a Wellcome Trust Senior Fellow in Clinical Science (WT098476MA). H. Yébenes was supported by a postdoctoral contract by the RTICC, and C. Abarrategui-Garrido was supported by the "Fundación de Investigación Biomédica del Hospital Universitario La Paz.”

Received for publication December 18, 2012, and accepted in revised form March 6, 2013.

Address correspondence to: Santiago Rodríguez de Córdoba, Centro de Investigaciones Biológicas, Ramiro de Maeztu 9, 28040 Madrid, Spain. Phone: 34.918373112; Fax: 34.915360432; E-mail: SRdeCordoba@cib.csic.es.
1. Jozsi M, Zipfel PF. Factor H family proteins and human diseases. Trends Immunol. 2008;29(8):380-387.

2. Perez-Caballero D, et al. Clustering of missense mutations in the C-terminal region of factor $\mathrm{H}$ in atypical hemolytic uremic syndrome. Am J Hum Genet. 2001;68(2):478-484.

3. Fritsche LG, et al. An imbalance of human complement regulatory proteins CFHR1, CFHR3 and factor $\mathrm{H}$ influences risk for age-related macular degeneration (AMD). Hum Mol Genet. 2010;19(23):4694-4704.

4. Heinen $S$, et al. Factor H-related protein 1 (CFHR-1) inhibits complement C5 convertase activity and terminal complex formation. Blood. 2009;114(12):2439-2447.

5. Hebecker M, Jozsi M. Factor H-related protein 4 activates complement by serving as a platform for the assembly of alternative pathway $\mathrm{C} 3$ convertase via its interaction with $\mathrm{C} 3 \mathrm{~b}$ protein. $\mathrm{J}$ Biol Chem. 2012;287(23):19528-19536.

6. Hellwage J, Jokiranta TS, Koistinen V, Vaarala O, Meri S, Zipfel PF. Functional properties of complement factor H-related proteins FHR-3 and FHR-4: binding to the $\mathrm{C} 3 \mathrm{~d}$ region of $\mathrm{C} 3 \mathrm{~b}$ and differential regulation by heparin. FEBS Lett. 1999;462(3):345-352.

7. McRae JL, et al. Human factor H-related protein 5 has cofactor activity, inhibits $\mathrm{C} 3$ convertase activity, binds heparin and C-reactive protein, and associates with lipoprotein. J Immunol. 2005;174(10):6250-6256.
8. de Cordoba SR, Tortajada A, Harris CL, Morgan BP. Complement dysregulation and disease: from genes and proteins to diagnostics and drugs. Immunobiology. 2012;217(11):1034-1046.

9. Hughes AE, Orr N, Esfandiary H, Diaz-Torres M, Goodship T, Chakravarthy U. A common CFH haplotype, with deletion of CFHR1 and CFHR3, is associated with lower risk of age-related macular degeneration. Nat Genet. 2006;38(10):1173-1177.

10. Gharavi AG, et al. Genome-wide association study identifies susceptibility loci for IgA nephropathy. Nat Genet. 2011;43(4):321-327.

11. Chen Q, et al. A novel hybrid CFHR2/CFHR5 gene develops MPGN II and provides insights into disease mechanism and therapeutic implications. Immunobiology. 2012;217:1131-1132.

12. Sanchez-Corral P, Gonzalez-Rubio C, Rodriguez de Cordoba S, Lopez-Trascasa M. Functional analysis in serum from atypical Hemolytic Uremic Syndrome patients reveals impaired protection of host cells associated with mutations in factor $\mathrm{H}$. Mol Immunol. 2004;41(1):81-84.

13. Goicoechea de Jorge E, et al. Structural findings in complement factor $\mathrm{H}$-related proteins unravel the pathogenesis of C3 glomerulopathy. Immunobiology. 2012;217:1205.

14. Fakhouri F, Fremeaux-Bacchi V, Noel LH, Cook HT, Pickering MC. C3 glomerulopathy: a new classification. Nat Rev Nephrol. 2010;6(8):494-499.

15. Gale DP, et al. Identification of a mutation in complement factor H-related protein 5 in patients of Cypriot origin with glomerulonephritis. Lancet. 2010;376(9743):794-801.

16. Zipfel PF, Jokiranta TS, Hellwage J, Koistinen V, Meri S. The factor $\mathrm{H}$ protein family. Immunopharmacology. 1999;42(1-3):53-60.

17. Skerka C, Zipfel PF. Complement factor H related proteins in immune diseases. Vaccine. 2008;26(suppl 8):I9-I14.

18. Venables JP, et al. Atypical haemolytic uraemic syndrome associated with a hybrid complement gene. PLoS Med. 2006;3(10):e431.

19. Heinen S, et al. De novo gene conversion in the RCA gene cluster (1q32) causes mutations in complement factor $\mathrm{H}$ associated with atypical hemolytic uremic syndrome. Hum Mutat. 2006; 27(3):292-293.

20. Tortajada A, Pinto S, Martinez-Ara J, Lopez-Trascasa M, Sanchez-Corral P, de Cordoba SR. Complement factor H variants 1890 and L1007 while commonly associated with atypical hemolytic uremic syndrome are polymorphisms with no functional significance. Kidney Int. 2012;81(1):56-63.

21. Licht C, et al. Deletion of Lys 224 in regulatory domain 4 of Factor $\mathrm{H}$ reveals a novel pathomechanism for dense deposit disease (MPGN II). Kidney Int. 2006;70(1):42-50.

22. Martinez-Barricarte R, et al. Human C3 mutation reveals a mechanism of dense deposit disease pathogenesis and provides insights into comple- 
ment activation and regulation. J Clin Invest. 2010;120(10):3702-3712.

23. Bomback AS, et al. Eculizumab for Dense Deposit Disease and C3 Glomerulonephritis. Clin J Am Soc Nephrol. 2012;7(5):748-756.

24. Vivarelli M, Pasini A, Emma F. Eculizumab for the treatment of dense-deposit disease. $N$ Engl J Med. 2012;366(12):1163-1165.

25. Daina E, Noris M, Remuzzi G. Eculizumab in a patient with dense-deposit disease. $N$ Engl J Med. 2012;366(12):1161-1163.

26. Radhakrishnan S, et al. Eculizumab and refractory membranoproliferative glomerulonephritis. NEngl JMed. 2012;366(12):1165-1166.

27. McCaughan JA, O'Rourke DM, Courtney AE. Recurrent dense deposit disease after renal transplantation: an emerging role for complementary therapies. Am J Transplant. 2012;12(4):1046-1051.
28. Fremeaux-Bacchi V, et al. Complement factor I: a susceptibility gene for atypical haemolytic uraemic syndrome. J Med Genet. 2004;41(6):e84.

29. Richards A, et al. Mutations in human complement regulator, membrane cofactor protein (CD46), predispose to development of familial hemolytic uremic syndrome. Proc Natl Acad Sci U S A 2003;100(22):12966-12971.

30. Goicoechea de Jorge E, et al. Gain-of-function mutations in complement factor B are associated with atypical hemolytic uremic syndrome. Proc Natl Acad Sci U S A. 2007;104(1):240-245.

31. Barrett MT, et al. Comparative genomic hybridization using oligonucleotide microarrays and total genomic DNA. Proc Natl Acad Sci U S A. 2004;101(51):17765-17770.

32. Alcorlo M, et al. Unique structure of $\mathrm{iC} 3 \mathrm{~b}$ resolved at a resolution of $24 \mathrm{~A}$ by $3 \mathrm{D}$-electron microscopy.
Proc Natl Acad Sci U S A. 2011;108(32):13236-13240. 33. Accardo P, Sanchez-Corral P, Criado O, Garcia E, Rodriguez de Cordoba S. Binding of human complement component $\mathrm{C} 4 \mathrm{~b}-$ binding protein $(\mathrm{C} 4 \mathrm{BP})$ to Streptococcus pyogenes involves the C4b-binding site. J Immunol. 1996;157(11):4935-4939.

34. Sanchez-Corral P, et al. Structural and functional characterization of factor $\mathrm{H}$ mutations associated with atypical hemolytic uremic syndrome. Am J Hum Genet. 2002;71(6):1285-1295.

35. Manuelian T, et al. Mutations in factor $\mathrm{H}$ reduce binding affinity to $\mathrm{C} 3 \mathrm{~b}$ and heparin and surface attachment to endothelial cells in hemolytic uremic syndrome. J Clin Invest. 2003;111(8):1181-1190.

36. Pickering MC, et al. Spontaneous hemolytic uremic syndrome triggered by complement factor $\mathrm{H}$ lacking surface recognition domains. J Exp Med. 2007;204(6):1249-1256 